\title{
Gastritis eosinofílica: a propósito de un caso
}

\author{
N. Madroñal Martín, E. GiL Sanzi \\ Médico residente åño de Medicina Familiar y Comunitaria. \\ 'Especialista en Medicina Familiar y Comunitaria. Centro de Salud Buenos Aires. \\ Área I. Madrid
}

\section{RESUMEN}

La gastritis eosinofílica es una enfermedad poco frecuente en la que existe una infiltración variable del tracto digestivo por eosinófilos maduros, apa rece eosinofilia periférica en un 70-80\% de los casos. Los síntomas de presentación más comunes son náuseas, vómitos, sensación de plenitud post prandial, dolor abdominal y pérdida de peso. Pre sentamos el caso de un varón de 50 años que acu dió en repetidas ocasiones a la consulta por epigastralgia intensa y vómitos. En el hemograma se detectó leucocitosis con eosinofilia periférica. El diagnóstico histológico tras gastroscopia fue de gastritis eosinofílica. El paciente permanece asin tomático tras instauración de tratamiento con cor ticoides orales.

Palabras clave: Gastritis eosinofílica. Epigas tralgia. Atención Primaria. Eosinofilia periférica.

\section{Eosinophilic gastritis: review of a case}

\begin{abstract}
Eosinophilic gastroenteritis is a rare disease in which there is a variable infiltration of the gas trointestinal tract by mature eosinophils, periphe ral eosinophilia is found in 70-80\% of cases. The most common symptoms are nausea, vomiting, ab dominal pain, abdominal fullness and weakness. We report the case of 50-year-old man with acute epigastric pain and vomiting. The peripheral-blood eosinophil count was high. The pathological diag nosis after endoscopic examination was eosinophi lic gastritis. The patient remains asymptomatic af ter oral steroids therapy.
\end{abstract}

Key words: Eosinophilic gastritis. Epigastric pain. Primary Health Care. Periferic eosinophilia.

\section{INTRODUCCIÓN}

La gastritis eosinofílica fue descrita por primera vez en $1937^{1}$. Es una enfermedad poco frecuente, se han registrado alrededor de 300 casos publicados en la literatura médica y no existen datos sobre su prevalencia. Aunque la enfermedad puede afectar a cualquier g rupo de edad, se suele presentar típica- mente entre la tercera y la quinta décadas de la vida $^{2}$. Su etiología es desconocida y se caracteriza por la infiltración variable del tracto digestivo por eosinófilos maduros. Respecto al patrón microscópico, la localización del infiltrado varía, siendo la variedad que afecta a la capa mucosa y submucosa la más frecuente $(57,5 \%)$. La clínica característica incluye dolor abdominal, náuseas, vómitos y dia-

Recepción: 17-04-02 
rrea. La afectación predominante de la capa muscular $(30 \%)$, produce obstrucción pilórica o suboclusión intestinal. El patrón más raro es el de afectación de la capa serosa $(12,5 \%)$ y se presenta como ascitis eosinofílica. Su diagnóstico definitivo es histológico ya que el único criterio diagnóstico válido es la demostración de un infiltrado eosinofílico en el tubo digestivo ${ }^{2}$. Macroscópicamente, el estómago y el intestino proximal son los más afectados habitualmente, pudiendo encontrarse comprometida cualquier porción del tracto digestivo por lo que el nombre más acertado de esta entidad es gastroenteritis eosinofílica (GEE).

\section{CASO CLÍNICO}

Varón de 50 años, con antecedentes personales médicos de hernia de hiato, alergia al polen y ácaros. Durante los dos últimos años había consultado en atención primaria de forma esporádica por molestias epigástricas. Estos episodios cedían con pautas cortas de antiácidos, antiH1 o bloqueantes de la bomba de protones, por lo que nunca se realizaron estudios complementarios hasta que consultó en cinco ocasiones consecutivas en el periodo de dos semanas por epigastralgia intensa que solía aparecer horas después de las comidas y vómitos abundantes de contenido alimentario, con sensación nauseosa y algún episodio diarreico sin productos patológicos. A la exploración destaca el aspecto malnutrido del paciente y el dolor a la palpación en epigastrio siendo el resto normal. Ante los síntomas tan intensos y recurrentes se decidió realizar un hemograma donde aparecieron 10.000 leucocitos/microlitro con $18,7 \%$ de eosinófilos, el resto del hemograma y la bioquímica fueron normales. Debido la persistencia de los síntomas tras veinte días de tratamiento con omeprazol y antieméticos, se decide realizar otras pruebas en nuestra consulta y mientras, derivamos al paciente a consulta especializada en gastroenterología para la realización de pruebas diagnósticas más específicas.

Ante la presencia de eosinofilia se realizó un frotis de sangre periférica que confirmó el número aumentado de eosinófilos (12.300 leucocitos con un $14,9 \%$ de eosinófilos) a expensas de elementos maduros. Este dato nos hizo descartar por el momento un síndrome hipereosinofílico idiopático (SHI) en el que suelen aparecen formas inmaduras en sangre periférica. Como pruebas de laboratorio habituales, el proteinograma, el análisis sistemático de orina y sedimento fueron normales. Con un coprocultivo y una muestra para parásitos en heces negativas, descartamos el origen parasitario de la enfermedad. Puesto que, ni en la anamnesis ni en la exploración, había datos de afectación de otros órganos o aparatos excepto el gastrointestinal, no se pidieron más estudios desde nuestra consulta. Las pruebas realizadas desde atención especializada consistieron en un tránsito esofagogastroduodenal que reveló vaciamiento gástrico lento, y una gastroscopia que mostró macroscópicamente patrón de gastritis crónica con erosiones gástricas y abundante contenido líquido en ayunas sin causa que lo justificara en la zona explorada. El test para $H$. pylori resultó negativo en las muestras recogidas, y la biopsia se informó como mucosa de tipo corporal con el patrón habitual de gastritis eosinofílica. Esta histológica junto con la exclusión de enfermedades parasitarias nos hizo llegar al diagnóstico de GEE.

En la consulta de gastroenterología se pautó tratamiento con prednisona oral a dosis de $40 \mathrm{mg}$ al día. Los controles sucesivos se realizaron en la consulta de Atención Primaria durante las tres siguientes semanas donde se observó que la sintomatología remitía rápidamente. El paciente ganó peso y comenzó a presentar mejor estado general, a partir de lo cual se espaciaron las visitas a nuestra consulta.

\section{DISCUSIÓN}

La gastritis eosinofílica es difícil de diagnosticar porque sólo el estudio histológico es concluyente ${ }^{3}$. El curso clínico habitual tiene carácter recidivante y los primeros brotes pueden pasar inadvertidos, como es el caso de nuestro paciente, el cual refería molestias epigástricas intermitentes de diferente intensidad durante los últimos dos años. Las crisis suelen ser benignas y autolimitadas, a no ser que se produzca presentación agresiva de la enfermedad, como úlceras que pueden llegar a ser gigantes ${ }^{4}$, formaciones que simulan neoplasias, o en el caso en que aparezca alguna complicación como perforación, obstrucción o ascitis.

El paciente presentado tiene 50 años en el momento del diagnóstico, y lleva al menos dos años con molestias epigástricas, por lo tanto entra dentro de la edad típica de presentación que se sitúa entre la tercera y la quinta década de la vida ${ }^{2,5}$. Además la edad del paciente nos permite descartar una gastroenteropatía alérgica infantil que se presenta antes de los 20 años ${ }^{6}$. Aunque la etiología es desconocida, se sospecha un componente alérgico a alimentos. Este dato sólo se aparece en algunos pacientes, pero sí se ha comprobado que el $50 \%$ de ellos presentan dermatitis atópica. Algunos de ellos padecen enfermedades de tipo alérgico tales como asma, rinitis alérgica o eccemas. En cualquier caso, el tratamiento con eliminación dietética ensayado en algunos pacientes no consiguió mejoría clínica ni anatomopatológica ${ }^{6}$. En el caso presentado existían pruebas de alergia realizadas previamente que fueron positivas para polen y ácaros del polvo, sin historia de alergias alimentarias. 
La enfermedad cursa en un $70-80 \%$ con eosinofilia periférica ${ }^{7}$. Su ausencia en algunos casos podría deberse a la infiltración masiva a nivel tisular. En nuestro caso existen cifras de eosinófilos bastantes superiores al 5\% del total de leucocitos, cifra a partir de la cual se considera patológica. Nos planteamos el diagnóstico diferencial con entidades que produzcan síntomas digestivos y puedan tener eosinofilia periférica (Tabla I). Entre ellas las infecciones parasitarias del tubo digestivo (amebiasis, esquistosomiasis, triquinosis, distomatosis, esquinococos, filarias, giardias y anisakis) son importantes por su frecuencia y tratamiento específico. Esto hace que descartar la presencia de huevos y parásitos en las heces de estos pacientes sea obligatorio ${ }^{8}$. El llamado síndrome hipereosinofílico idiopático (SHI) también merece mención especial. Sus criterios diagnósticos se especifican en la tabla II. Es una entidad muy infrecuente que predomina en sexo masculino en proporción 9/1 y afecta a individuos de edades comprendidas entre 20 y 50 años ${ }^{10}$. La eosinofilia puede alcanzar cifras muy elevadas (50.000 a 100.000 células/microlitro) y se observan a menudo formas inmaduras en sangre periférica ${ }^{8,9}$. Es una enfermedad con mal pronóstico donde predominan las manifestaciones hematológicas y cardiacas. El corazón se afecta en el $90 \%$ de los casos y la enfermedad cardiovascular suele ser la causa de

\section{Tabla I}

DIAGNÓSTICO DIFERENCIAL DE LA GEE CON ENTIDADES QUE PUEDEN CURSAR CON SIINTOMAS GASTROINTESTINALES Y EOSINOFILIA PERIFÉRICA

Parasitosis

Síndrome hiperoesinofílico idiopático

Mastocitosis sistémica

Enfermedad inflamatoria intestinal

Enfermedad celíaca

Linfoma intestinal muerte $^{10}$. Puede afectar otros órganos y tejidos como pulmón, sistema nervioso central y periférico $\mathrm{y}$ tracto digestivo. Aunque en el caso presentado las cifras de eosinófilos en sangre entran en el rango de SHI, en el frotis de sangre periférica no se vieron formas inmaduras y el paciente no presenta síntomas ni signos fuera del sistema digestivo.

La mayoría de los autores consideran el SHI una entidad diferente a la GEE, pero otros sugieren que la GEE podría ser una manifestación aislada del SHI, así como el granuloma eosinófilo sería la forma localizada de la misma entidad ${ }^{9}$.

Según algunos estudios, un $20 \%$ de enfermos con GEE no presentan eosinofilia periférica ${ }^{11,}$ por eso cabe realizar el diagnóstico diferencial con enfermedades que sólo presentan infiltración por eosinófilos en la pared del tubo digestivo (Tabla III). Como ya se ha comentado, la demostración anatomopatológica del infiltrado eosinofílico en el tubo digestivo es imprescindible y constituye, en ausencia de una etiología clara, el único criterio diagnóstico válido para definir la enfermedad ${ }^{6}$. Al afectarse cualquier tramo del tubo digestivo se requiere una panendoscopia ${ }^{6,12}$ y la obtención de múltiples biopsias (se establece un mínimo de seis $)^{8}$, ya que la enfermedad no suele ser continua sino parcheada. Los hallazgos microscópicos principales son edema e infiltración eosinofílica de la pared intestinal; este hecho puede verse también en otras patologías (Tabla III); pero en ellas, este infiltrado no suele ser tan intenso como en la GEE (se consideran diagnósticos más de 60 eosinófilos por campo de diez aumentos) ${ }^{8}$ y se acompañan de importante presencia de células mononucleares que están ausentes en la GEE.

En la gastritis eosinofílica existen tres patrones posibles de infiltración del tracto digestivo ${ }^{5,7,8}$ :

1. Afectación de la capa mucosa que es el más frecuente. Cursa con náuseas, vómitos y dolor

\section{Tabla II}

DIAGNÓSTICO DIFERENCIAL DE GEE CON ENTIDADES EN LAS QUE EXISTE INFILTRACIÓN EOSINOFÍLICA DEL APARATO DIGESTIVO

Parasitosis

Granuloma eosinófilo

Inflamación crónica

Enfermedad celíaca

Linfoma Hodgkin

Enfermedad de Crohn

Síndrome de Churg-Strauss

Carcinoma 
abdominal más intenso después de las comidas. Si afecta parte del intestino delgado, puede haber diarrea y malabsorción.

2. Alteración de la capa muscular, segundo patrón en frecuencia, que produce engrosamiento y rigidez de la muscular propia. Típicamente se manifiesta con signos y síntomas de estenosis pilórica intermitente $\mathrm{o} / \mathrm{y}$ obstrucción intestinal incompleta.

3. La infiltración de la capa serosa es la menos frecuente y cursa con ascitis exudativa.

Las muestras tomadas en el caso presentado procedían de mucosa gástrica de tipo corporal y tenía signos de gastritis aguda con muchos eosinófilos maduros. Este es el patrón característico de la enfermedad, y explica el dolor, náuseas y vómitos postprandiales. Podemos suponer cierta lesión mucosa en intestino delgado, lo explicaría en parte el aspecto malnutrido del paciente y los episodios de diarrea ocasionales. Hay algunos datos que indican cierto grado de lesión muscular especialmente la zona antropilórica: tanto en la endoscopia como en el tránsito intestinal realizados se aprecia vaciamiento gástrico lento sin ninguna causa macroscópica que lo justifique.

La GEE es una entidad benigna con buen pronóstico en general, por eso en los casos con síntomas leves y esporádicos se puede adoptar una actitud expectante tratando de retrasar el comienzo del tratamiento ya que no está exento de efectos secundarios. El tratamiento de elección son los corticoides por vía oral que inhiben la producción de IL5, implicada en la quimiotaxis de los eosinófilos, y disminuyen la vida media de los mismos. La dosis de prednisona oral es $20-40 \mathrm{mg}$ en una sola dosis al día ${ }^{2,5,13}$ durante una o dos semanas, en las que obtiene una respuesta muy rápida controlándose los síntomas digestivos satisfactoriamente. Si no se produce una respuesta rápida a este tratamiento se debe dudar del diagnóstico de GEE. El objetivo tras la mejoría es disminuir la dosis lenta y progresivamente hasta suspender el tratamiento. Algunas veces no es posible la retirada del fármaco completamente ya que reaparecen los síntomas digestivos, estos pacientes precisan dosis pequeñas y mantenidas de corticoides ${ }^{6,8}$. Algunos estudios refieren que las cifras de eosinófilos se normalizan al cabo de un año de tratamiento. En el caso presentado el paciente respondió inicialmente a $40 \mathrm{mg} /$ día de prednisona con remisión de los síntomas y ganancia de peso.

La mortalidad por GEE es excepcional y suele estar más relacionada con complicaciones quirúrgicas que con la propia enfermedad ${ }^{6}$. En algunos casos la evolución de estos pacientes a pesar de un diagnóstico y un tratamiento correctos es tórpido y se han descrito casos de abdomen agudo con perforación intestinal debida a la enfermedad pero son los menos frecuentes. Hay algunos casos en la bibliografía de pacientes intervenidos de urgencia con la sospecha de apendicitis aguda o de abdomen agudo sin un diagnóstico claro donde se podría haber evitado la operación al pautar un tratamiento correcto. Incluso hemos localizado en la literatura un caso en el que se realizó una antrectomía distal por una úlcera gigante que, tras el análisis histológico, resultó ser GEE y respondió después espectacularmente al tratamiento con corticoides ${ }^{4}$.

Otros tratamientos con antihistamínicos y la retirada de los posibles alimentos desencadenantes de alergias son ineficaces. Los estudios que hay sobre el uso de estabilizadores de la membrana de los mastocitos como el ketotifeno y el cromoglicato sódico no son concluyentes. Lo que sí parece es que la asociación de estos fármacos a los corticoides permitiría reducir la dosis de éstos en pacientes que los precisan para mantenerse en remisión clínica. Más recientemente se ha hablado del Montelukast como tratamiento para la GEE, pero no se tienen datos de hasta que punto este fármaco sería capaz de mantener remisiones sin estar asociado con corticoides $^{14}$.

\section{CONCLUSIÓN}

La GEE es una entidad con curso crónico que precisa de un alto nivel de sospecha para su diagnóstico. Los síntomas suelen ser molestias digestivas comunes, autolimitadas y poco importantes en su inicio, sin embargo, la progresión de la enfermedad puede terminar en la aparición de complicaciones graves o llevarnos a realizar intervenciones diagnósticas y/o presumiblemente terapéuticas agresivas que, lejos de mejoras los síntomas, van a empeorar la situación del paciente. El médico de Atención Primaria, a través del seguimiento clínico a lo largo del tiempo del paciente, se encuentra en una situación privilegiada para sospechar precozmente este tipo de patología que se presenta con sintomatología vaga pero recurrente. Todo ello permitiría un diagnóstico y tratamiento eficaz en un plazo menor de tiempo.

\author{
CORRESPONDENCIA: \\ Esther Gil Sanz \\ Equipo de Atención Primaria Portazgo \\ Centro de Salud Buenos Aires \\ C/ Pío Felipe con c/ Boada, s/n \\ 28038 Madrid
}




\section{Bibliografía}

1. Kaijer R. Zur Kenntnis der Allergischen Affektionen des Verdauungskanals Von Stanpunkt des Chirurgan aus. Arch Klin Chir 1937; 36: 188.

2. Ruiz Montes F, René Espinet J, Rubio Caballero M. Gastroenteritis eosinofílica: Revisión de los casos publicados en España y comparación con la literatura extranjera. Rev Esp Enferm Dig 1992; 81: 270-9.

3. Cazaña JL, Blanco Guerra C, Crespo JF, Contreras J, Martínez Alzamora F. Gastritis Eosinofílica: resolución espontánea. Rev Esp Enferm Dig 1996; 82: 51-2.

4. Scolapio JS, Kenneth DeVault MD, Wolfe JT. Eosinophilic gastroenteritis presenting as a giant ulcer. AJG 1996; 91: 804-5.

5. Case records of the Massachussets General Hospital. Weekly clinicopathological exercices. N Engl J Med 1992; 326: $1342-9$

6. Remacha Tomey B, Palau A, Colom J, Ripollés V. Gastroenteritis eosinofílica. An Med Interna (Madrid) 1998; 15: 554-9.

7. Valley NJ, Shorter RG, Phillips SF, Zinsmeister AR. Eosinophilic gastritis: a clinicopathological study of patients with disease of the mucosa, muscle layer and subserosal tissues. Gut 1990; 31: 54-8.
8. Rodriguez Leal C, Tinoco Racero I, Téllez Pérez F, Girón González JA. Gastroenteritis eosinofílica. Medicine 2000; 8: $238-41$.

9. Santana García S, Fuertes Martín A, Guerrero Peral AL, Aparicio Velasco J, Jiménez López A. Gastroenteritis eosinofílica y tromboembolismo pulmonar. ¿Asociación casual? An Med Interna (Madrid) 1995; 12: 73-5.

10. Loyola I, Ariza A. Varón de 51 años con dolor abdominal, parestesias en extremidades, fiebre y eosinofilia. Med Clin (Barc) 1993; 101: 28-35.

11. Cervantes $\mathrm{X}$, Vidal A. Dolor epigástrico crónico en un varón de 18 años. Med Clin (Barc) 1997; 108: 750-6.

12. Chuan-Mo L, Chi-Sin C, Pang-Chi C, Deng-Yn L, I-Shyan $\mathrm{S}$, Chia-Siu W, et al. Eosinophilic Gastroenteritis: 10 years experience. Am J Gastroenterol 1993; 88: 70-3.

13. Soriano Izquierdo A, Sans Cuffi M, Elizalde Frez JI, Bessa Caserras X. Gastritis y gastropatías. Medicine 2000; 8: 62-8.

14. Bastida Eizaguirre M, Iturbe Ortiz de Urbina R, Arto Urzainqui MJ, Muñecas Ibarrechebea J, Beltrán de Heredia P. Gastroenteritis eosinofílica en un paciente afectado de osteodistrofia hereditaria de Albright. An Esp Pediatr 2002; 56: $576-8$ 